\title{
Controlling second-harmonic generation at the nanoscale with monolithic AIGaAs-on- AIOx antennas
}

\author{
L Carletti ${ }^{1}$, D Rocco ${ }^{1}$, A Locatelli ${ }^{1}$, C De Angelis ${ }^{1}$, V F Gili ${ }^{2}$, M Ravaro ${ }^{2}$, \\ I Favero ${ }^{2}$, G Leo $^{2}$, M Finazzi $^{3}$, L Ghirardini ${ }^{3}$, M Celebrano $^{3}$, G Marino $^{4}$ and \\ A V Zayats ${ }^{4}$ \\ ${ }^{1}$ Department of Information Engineering, University of Brescia, Via Branze 38, 25123 Brescia, Italy \\ ${ }^{2}$ Matériaux et Phénoménes Quantiques, Université Paris Diderot-CNRS, 10 rue A. Domon et L. Duquet, \\ 75013 Paris, France \\ ${ }^{3}$ Department of Physics, Politecnico di Milano, Piazza Leonardo Da Vinci 32, 20133 Milano, Italy \\ ${ }^{4}$ Department of Physics, King's College London, Strand, London, WC2R 2LS, United Kingdom
}

Received 27 September 2016, revised 16 December 2016

Accepted for publication 3 January 2017

Published 16 February 2017

\begin{abstract}
We review recent achievements in the field of nanoscale nonlinear AlGaAs photonics based on all-dielectric optical antennas. After discussing the motivation and main technological challenges for the development of an AlGaAs monolithic platform for $\chi^{(2)}$ nonlinear nanophotonics, we present numerical and experimental investigations of the second-order nonlinear response and physical reasons for high efficiency of second-order nonlinear interactions in the AlGaAs nanoantennas. In particular, we emphasize the role of the dipolar resonances at the fundamental frequency and the multipolar resonances at the second harmonic wavelength. We also discuss second-harmonic generation directionality and show possible strategies to engineer the radiation pattern of nonlinear antennas.
\end{abstract}

Keywords: optical antennas, metamaterials, second harmonic generation

\section{Introduction}

In recent years, light manipulation by means of nanoscale structures has attracted enormous interest due to its impact on modern photonic technologies [1-6]. Not only might nanoscale objects facilitate integration of various linear and nonlinear functionalities in nanophotonic circuits, but they also tremendously improve miniaturization allowing one to replace bulky components, which typically rely on free-space optics. The opportunity of fabricating ultrathin devices compatible with planar manufacturing technology enhances this drive. Many devices and functionalities have been demonstrated through the exploitation of metallic and/or dielectric nanoparticles for different applications. Those based on metallic nanostructures exhibiting plasmonic resonances have already had a significant impact in diverse application areas such as sensing, nanomedicine, telecommunications, quantum optics, and photovoltaics [7-13]. However, plasmonic nanostructures have an inherent weakness related to the Ohmic losses at optical frequencies, which limit their usefulness in certain applications. For example, the losses generate Joule heating that could hamper the use of plasmonics in some applications such as sensing where a temperature increase, if not mitigated, could alter or even damage the properties of a monitored analyte [14-16]. In dielectric materials, this problem is less severe given their relatively smaller optical losses in the visible and near-infrared wavelength range.

At the same time, nanostructures made of dielectrics with a relatively high refractive index are capable of tight light confinement [17-20]. For these reasons, metal-less nanophotonics employing high-refractive index dielectrics and semiconductors, such as silicon, germanium, tellurium, gallium arsenide, aluminium gallium arsenide, gallium phosphide, are now emerging as a promising alternative to plasmonic nanostructures for nanophotonic applications [21]. 
In particular, significant progress has been achieved with high permittivity dielectric nanoparticles in a low refractive index background, which exhibit negligible dissipative losses and strong Mie resonances in the visible and near-infrared spectral ranges. These new systems may be employed in many applications such as directional scattering and emission, surface enhanced vibrational spectroscopy, and flat metasurfaces for phase-front engineering. Depending on a particular application, one of the two approaches-metal or metal-less nanophotonics-may have beneficial properties [22-29]. For example, in metallic nanoparticles the electric field is strongly confined close to the metal surface, enhancing light-matter interaction close to the surface, whereas in dielectric nanoparticles the electric field of the resonant modes penetrates deep inside their volume, enhancing light-matter interaction with the bulk of a nanoparticle [30-40]. This encourages the use of all-dielectric nanoantennas for strengthening the nonlinear optical response from the nanoparticles and some promising results have already been achieved in the framework of semiconductor nanoparticles.

Semiconductors exhibit quite strong second- and/or thirdorder nonlinear optical susceptibilities compared to other materials such as insulators (e.g., $\mathrm{SiO}_{2}, \mathrm{TiO}_{2}$ ); however, typical values of the nonlinear susceptibility are small, requiring light fields comparable to the characteristic atomic field strength, and high pump intensities are often needed to obtain nonlinear signals that might be of practical use in applications. Since the power of the nonlinearly generated second (third) harmonic frequency scales with the fourth (sixth) power of the fundamental field strength, the possibility to confine the electromagnetic energy into tiny volumes in nanoparticles is very important to enable the downscaling of the required optical powers [23]. Using a silicon platform, third-harmonic generation (THG) in silicon-on-insulator (SOI) nanoantennas has been recently investigated. In the pioneering works [41, 42], it was experimentally confirmed that a strong enhancement of THG (up to 100 over the case of a silicon slab) can be achieved in isolated silicon nanodisks by optically exciting at frequencies near the magnetic dipolar resonance. Another experimental demonstration showed an increase of two-photon absorption (TPA) by almost two orders of magnitude in hydrogenated amorphous silicon nanodisks with respect to an unstructured silicon film [31], allowing one to achieve strong self-modulation of femtose-cond pulses, resulting in an important step towards active photonic devices [43]. Although these outcomes clearly illustrate the potential of all-dielectric nanoparticles for non-linear nanophotonic applications, they are limited by the inherent constraints of the SOI platform in which they have been obtained.

Despite the spectacular achievements of silicon technology, only $\chi^{(3)}$ effects have been observed since, because of its centrosymmetric crystal structure, silicon does not exhibit bulk quadratic optical nonlinearity. In order to overcome this limitation, non-centrosymmetric materials have recently been studied such as AlGaAs alloys. $\mathrm{Al}_{\mathrm{x}} \mathrm{Ga}_{1-\mathrm{x}}$ As is a popular III-V material for integrated nonlinear optics [44, 45] because it combines some properties that it shares with $\mathrm{Si}$ (a similar refractive index in the near infrared, a comparatively high $\chi^{(3)}$ nonlinear index, and a good thermal conductivity) with additional key advantages including (1) a direct band gap for $\mathrm{Al}$ molar fraction below 0.45 that increases with the $\mathrm{Al}$ content enabling TPA-free operation at $1.55 \mu \mathrm{m}$ [46], (2) a high nonresonant second-order susceptibility $\left(d_{14} \approx 100 \mathrm{pm} \mathrm{V}^{-1}\right.$ for GaAs in the near infrared), and (3) a broad spectral window of transparency in the mid-infrared (up to $17 \mu \mathrm{m}$ ). In this context, the hybridization of AlGaAs with SOI and AlGaAs-based monolithic platforms have a sig-nificant potential for $\chi^{(2)}$ nonlinear nanophotonics.

Here we overview the recent results obtained in the field of second-order nonlinear effects in AlGaAs nanoantennas. The paper is organized as follows. Section 2 presents the fabrication of AlGaAs nanoparticle-based monolithic platform for $\chi^{(2)}$ nonlinear nanophotonics. In section 3, the linear optical properties of these nanoparticles are described which can be considered as nanoscale resonators. Obviously, efficiencies of nonlinear processes are significantly increased through the enhancement of local fields at the resonances, allowing for nonlinear optical components to be scaled down in size and offering exclusive prospects for all-optical light control at the nanoscale. In section 4, we compare the theoretical predictions with recently obtained experimental results, particularly emphasizing the role played by the dipolar resonances at the fundamental frequency and the multipolar resonances at the second harmonic frequency. In section 5, we then discuss directionality issues and show some possible strategies to engineer the radiation pattern of nonlinear nanoantennas, using the properties of the funda-mental frequency field (i.e. polarization state and propagation direction) as tuning parameters. In section 6, we discuss a more general framework which includes sum and difference frequency generation and optical parametric amplification. Finally, an outlook for this young and active research field of secondorder nonlinear photonics in dielectric nanoantennas is given.

\section{Fabrication and technological challenges}

The basic requirement for fabricating AlGaAs monolithic nanoantennas is that they are surrounded by a low refractiveindex medium compatible with nanophotonic technology. Two options are available: air (refractive index $n=1$ ) or aluminum oxide ( $n \approx 1.6$ ). In the former case, the nanoantenna can be more efficiently decoupled from the underlying GaAs wafer, but it has to be suspended with photonic-crystallike fabrication techniques. In the latter case, one can use the wet oxidation of an underlying AlAs layer [33], resulting in a better thermal dissipation, at the price of a slightly smaller optical confinement due to the lower index contrast between the nanostructure $\left(n \approx 3.2\right.$ for $\mathrm{Al}_{0.18} \mathrm{Ga}_{0.82} \mathrm{As}$ in the near IR) and its environment. A few hybrid schemes are also possible, based on the transfer of epitaxial AlGaAs on silica [47] or benzocyclobutane (BCB) [48], or on the CMOS-compatible wafer bonding of an $\mathrm{AlGaAs}$ heterostructure on silicon oxide [49]. However, they fall out of the scope of this review, which 

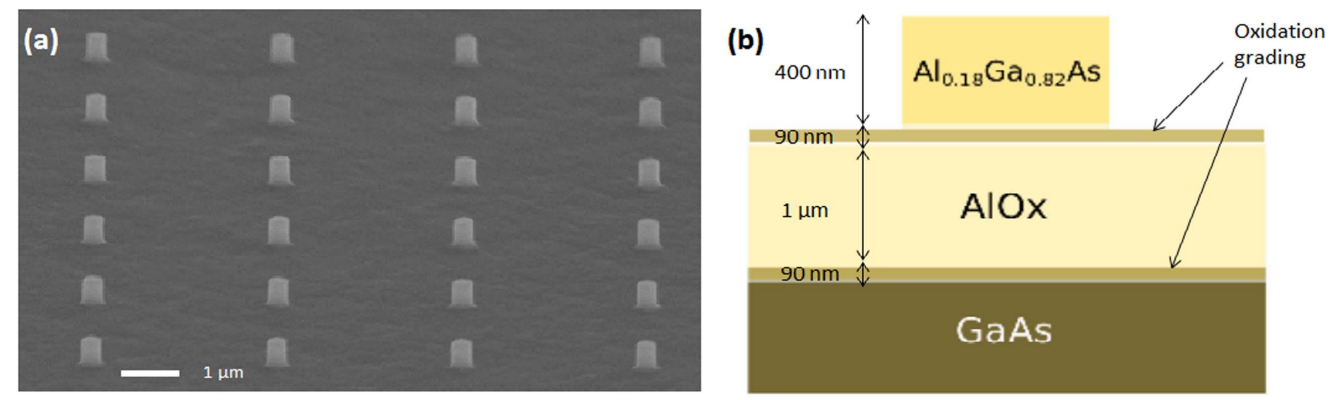

Figure 1. Monolithic AlGaAs-on-AlOx nanoantennas: (a) scanning electron microscope image of the nanoantenna array; (b) schematics of a single nanoantenna on a substrate. Reproduced from [32]. CC BY 4.0.

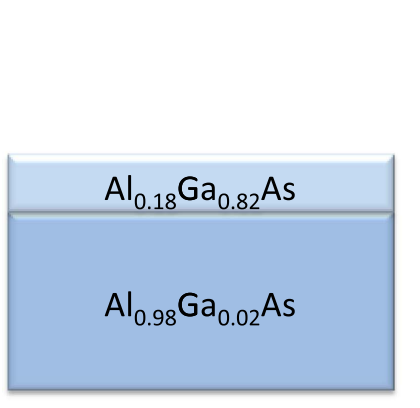

a)

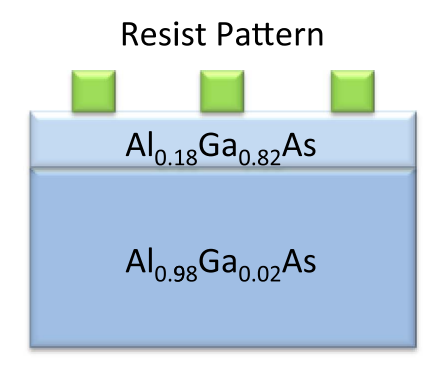

b)

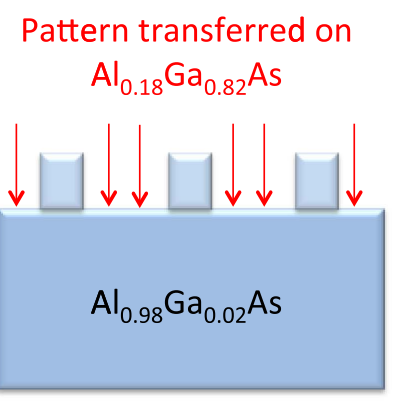

c)

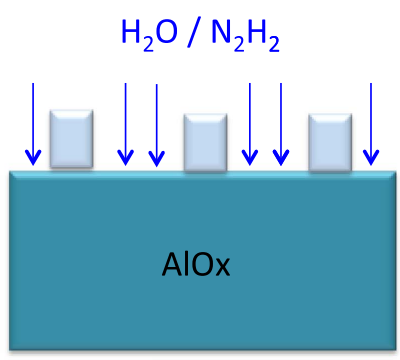

d)

Figure 2. Fabrication steps of optical nanoantennas: (a) Epitaxial growth; (b) Electron beam lithography; (c) Reactive ion etching; (d) Selective oxidization.

focuses on monolithic devices that lend themselves to the with varying aluminum molar fraction. In order to obtain an array perspective of full optoelectronic integration with optical of nanocylinders shown in figure 1(a), circles were pat-terned sources and detectors. Finally, because of its relative simpli- using scanning electron microscope lithography, with radii city, the AlGaAs-on-oxide is the only monolithic nanoan- between $175 \mathrm{~nm}$ and $225 \mathrm{~nm}$ equally spaced by $3 \mu \mathrm{m}$. Then the tenna with second-order nolinearity demonstrated to samples were dry-etched with non-selective ICP-RIE with date $[33,34]$. $\mathrm{SiCl}_{4}$ :Ar chemical treatment. The etching depth of $400 \mathrm{~nm}$,

Until recently, a development of an AlGaAs photonic controlled by laser interferometer, defined the nanocylinders and platform was hindered by the difficulty of fabricating mono- revealed the AlAs layer. The etched sample was then oxidized at lithic shallow waveguides and cavities as in the silicon-on- $390{ }^{\circ} \mathrm{C}$ for $30 \mathrm{~min}$ in an oven equipped with in situ optical insulator (SOI) system, and in particular by the shortcomings of monitoring, under a precisely controlled water vapor flow with a the wet selective oxidation of $\mathrm{AlGaAs}$ epitaxial layers. The $\mathrm{N}_{2}: \mathrm{H}_{2}$ gas carrier. After oxidation, each $\mathrm{Al}_{0.18} \mathrm{Ga}_{0.82} \mathrm{As}$ nanolatter was discovered in 1990 and results in a non-stoichio- cylinder lies upon a uniform AlOx substrate (figure 1(b)), whose metric alumina $\left(\mathrm{AlO}_{\mathrm{x}}\right.$, also called $\left.\mathrm{AlOx}\right)$ with optical and low refractive index enables sub-wavelength optical confine-ment electrical properties similar to $\mathrm{SiO}_{2}$ [45]. The use of $\mathrm{AlOx}$ layers in the nanocavity by total internal reflection. As expected from the thinner than $100 \mathrm{~nm}$ is standard in VCSEL technology [50] and strong selectivity of oxidation kinetics relative to alu-minum has also results in the demonstration of an optical parametric molar fraction, no post-oxidation cross-section reduction has been oscillator in an AlGaAs waveguide [51]. However, the observed by transmission electron microscopy on a thin slice of fabrication of high-quality $\mu$ m-thick $\mathrm{AlOx}$ optical sub-strates is $\mathrm{Al}_{0.18} \mathrm{Ga}_{0.82} \mathrm{As}$ nanopillars. Note also that $\mathrm{Al}_{0.18} \mathrm{Ga}_{0.82} \mathrm{As}$ critical because the selective oxidation of AlGaAs optical oxidation is negligible under these experimental conditions. substrates induces a strong contraction of the oxide. This While initially the fabrication conditions with temperature cases typically results in important optical losses in integrated below $400{ }^{\circ} \mathrm{C}$ and $\mathrm{Al}$ content $\leqslant 0.45$ have never been considered, photonic devices, due to defects at the interface between AlOx our approach shows an extremely shallow surface oxidation and the adjacent crystal [52].

which may provide an upper limit to the optical quality of very-

Nanoantennas are typically grown by molecular-beam-epi- high-Q $\left(10^{5}\right)$ microcavities; however, its impact here is negligible taxy on [100] non-intentionally doped GaAs wafer, with a $400 \mathrm{~nm}$ because the Q-factor of our sub-wavelength nanoantennas is layer of $\mathrm{Al}_{0.18} \mathrm{Ga}_{0.82} \mathrm{As}$ on top of an aluminum-rich considerably smaller.

substrate, to be oxidized at a later stage. In order to improve the eventual optical quality of the interface between $\mathrm{AlOx}$ and the steps, it is apparent that deep etching into the $\mathrm{Al}_{0.98} \mathrm{Ga}_{0.02} \mathrm{As}$ adjacent crystalline layers, such substrate consists of $1 \mu \mathrm{m}$-thick layer (step c) is possible to increase the lateral confinement of $\mathrm{Al}_{0.98} \mathrm{Ga}_{0.02} \mathrm{As}$ layer sandwiched between two transition regions the nanoantenna optical modes. While this is indeed the 


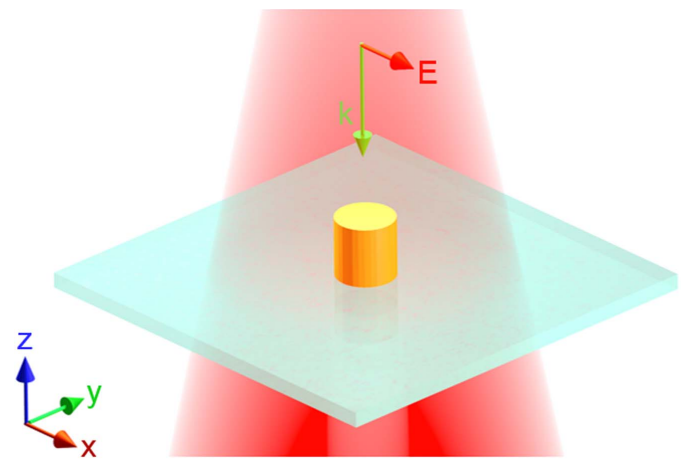

Figure 3. Conceptual representation of scattering from an AlGaAs nanocylinder on a low-refractive index substrate. The red pump beam excites the cylindrical nanoantenna. The incident field is shown with the arrows representing $\mathrm{E}$ and $\mathrm{k}$ in the $x-z$ plane.

approach reported in [34], for nonlinear optical applications we find it more appropriate to let the crystalline nanocylinders to sit on a uniform AlOx layer, which is a better heat sink than a nanoscopic $\mathrm{AlOx}$ pedestal.

\section{Linear properties of the nanoantennas for SHG enhancement}

The design of antennas for enhancing the second-order nonlinear optical response requires information on their basic properties such as resonant frequencies, bandwidth, field distribution and radiation diagrams at fundamental and second harmonic $(\mathrm{SH})$ frequencies. Different strategies can be employed to model such nanoantennas. Once the optical response of a nanoparticle is calculated numerically, a very powerful approach to interpret the linear and nonlinear scattering resorts to multipole decomposition [53]. Adjusting phase and amplitude of the different multipole contributions provides a robust tool to engineer the radiation patterns at the desired operating frequencies.

The scattering spectra of $\mathrm{Al}_{0.18} \mathrm{Ga}_{0.82} \mathrm{As}$ cylinders at near-IR wavelengths were simulated using finite element method simulations in COMSOL [32]. The pump can be chosen as a plane wave or a light field with a more complex structure (figure 3 ). Let us first recall what has been reported using either plane waves or focused Gaussian beams, with propagation along the cylinder axis $(z)$ and the electric field, $\mathbf{E}$, along the $x$ axis.

To account for wavelength dependence of the refractive index of $\mathrm{Al}_{0.18} \mathrm{Ga}_{0.82} \mathrm{As}$, the analytical model proposed in [54] can be used, which was derived from comparison with experimental measurements. We define the extinction as $Q_{\mathrm{e}}=Q_{\mathrm{s}}+Q_{\mathrm{a}}$, where $Q_{\mathrm{s}}=C / \pi r^{2}$ and $Q_{\mathrm{a}}=A / \pi r^{2}$ are the scattering and absorption, respectively, with $C$ being the scattering cross-section, $A$ being the absorption crosssection, and $r$ being the cylinder radius. A plot of $Q_{\mathrm{e}}$ in the near-infrared spectral region calculated for a cylinder with radius $r=187 \mathrm{~nm}$ and height $h=400 \mathrm{~nm}$ on a substrate with a refractive index of 1.6 is presented in figure 4(a). We can observe two resonant peaks for wavelengths shorter than $1500 \mathrm{~nm}$ while, for longer wavelengths, the scattering efficiency monotonically decreases. In particular, the sharpest and strongest resonance observed at $\lambda=1475 \mathrm{~nm}$ is mainly due to a MD resonance, as already discussed in the literature [55-57]. The broader resonance at $\lambda=1200 \mathrm{~nm}$ has electric dipole character. To understand the SHG properties of such nanopillars for a fundamental frequency (FF) in the spectral range indicated in figure 4(a), we calculated the scattering efficiency at the corresponding $\mathrm{SH}$ wavelengths shown in figure 4(b). Since, in this spectral region the antenna has a multipolar response, it has been calculated using exciting dipoles at different random positions that allow exciting all dark and bright modes [29]. By comparing figures 4(a) and (b), we can see that, as expected, in the longer wavelength range, where the FF frequency sits, the scattering is dominated by lower-order multipoles, while in the $\mathrm{SH}$ frequency range a multitude of scattering contributions due to higher-order multipoles are responsible for the observed features. It is also important to stress the presence of the long absorption tail below approximately $725 \mathrm{~nm}$, which determines the minimum possible wavelength at which SHG can be highly enhanced via the bulk $\chi^{(2)}$ of $\mathrm{Al}_{0.18} \mathrm{Ga}_{0.82} \mathrm{As}$.

\section{SHG from AIGaAs nanoantennas: efficiency considerations}

SHG from AlGaAs nanoantennas has been recently investigated in [32-34, 48, 58]. In striking contrast with typical plasmonic materials, since AlGaAs is a non-centrosymmetric material, there is a strong volume contribution to the overall efficiency of the SHG process. Moreover, the only non-zero element of the second-order nonlinear susceptibility $\chi^{(2)}{ }_{\text {iik }}$ of $\mathrm{Al}_{0.18} \mathrm{Ga}_{0.82} \mathrm{As}$ has $i \neq j \neq k$, with $\mathrm{d}_{14}=\chi^{(2)} / 2 \approx 100 \mathrm{pm} \mathrm{V}^{-1}$ [59].

In order to quantify the potential of AlGaAs nanocylinders for nonlinear nanophotonics, one can model the SHG phenomenon with COMSOL by using the nonlinear polarization induced by $\chi^{(2)}$ as a source in the simulations, and then define the SHG efficiency as:

$$
\eta_{S H G}=\frac{\int_{A} \vec{S}_{S H} \cdot \hat{n} \mathrm{~d} A}{I_{0} \pi r^{2}}
$$

where $\vec{S}_{S H}$ is the Poynting vector of the SH field, $\hat{n}$ is the unit vector normal to a surface $A$ enclosing the antenna, and $I_{0}$ is the incident field intensity $\left(I_{0}=1 \mathrm{GW} \mathrm{cm}^{-2}\right.$ in the simulations). The calculated SHG efficiency for a cylinder with $r=187 \mathrm{~nm}$ and $h=400 \mathrm{~nm}$ on a substrate of $n=1.6$ is shown in figure 5(a). As can be seen in the figure, there are three different SHG peaks. The corresponding fundamental frequencies fall inside the broad MD resonance peak and, thus, the associated field enhancement improves the efficiency of the nonlinear process. Inside this broadband resonance, the frequency selection rules for the maximum $\mathrm{SH}$ signal are determined by the resonances of the nanoantennas at shorter 

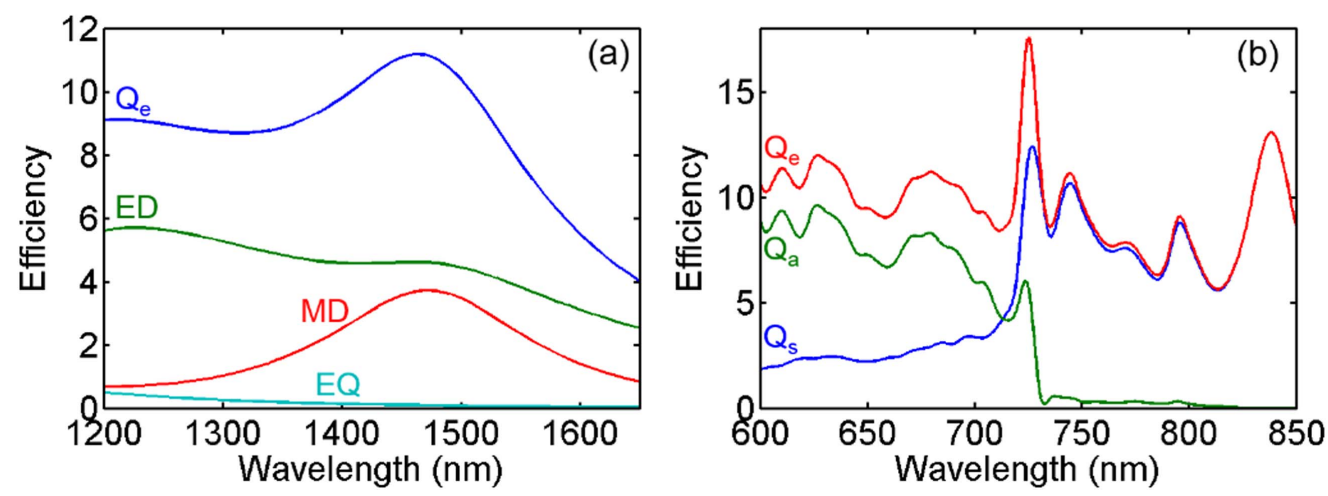

Figure 4. Linear spectral response simulated for the nanoantennas in figure $1(r=187 \mathrm{~nm}$ and $h=400 \mathrm{~nm})$. (a) Extinction efficiency in the fundamental wavelength range; the relevant lowest-order multipole contributions are magnetic dipole (MD), electric dipole (ED) and electric quadrupole (EQ). (b) Scattering $\left(Q_{\mathrm{s}}\right)$, absorption $\left(Q_{\mathrm{a}}\right)$ and extinction $\left(Q_{\mathrm{e}}\right)$ efficiencies in the $\mathrm{SH}$ wavelength range.
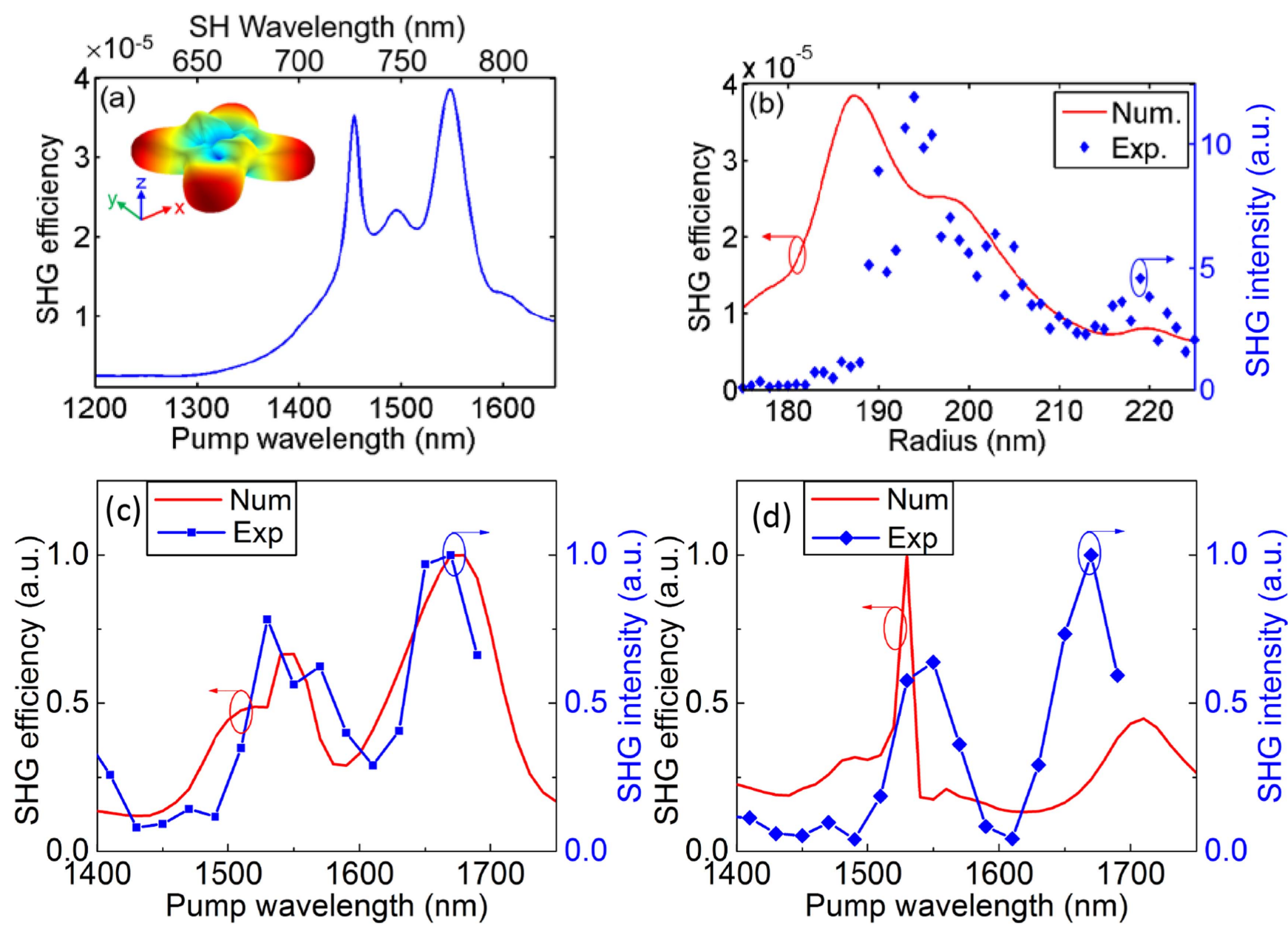

Figure 5. (a) Simulated SHG efficiency as a function of fundamental wavelength for a nanoantenna of radius $r=187 \mathrm{~nm}$. (Inset) A radiation pattern of the emitted second harmonic light for a fundamental wavelength $\lambda$ corresponding to $r / \lambda=0.133$. (b) Numerical (continuous line) and experimental (diamonds) dependences of SHG on the radius of the AlGaAs nanoantennas for a fundamental wavelength of $1550 \mathrm{~nm}$. (c) and (d) Measured (red lines) and simulated (blue lines) SHG spectra from the nanoantennas of a radius of $225 \mathrm{~nm}$ for co-polarised (c) and (d) cross-polarised pump/SHG configurations. The nanoantennas height in (a-d) is $400 \mathrm{~nm}$.

wavelengths; in figure 5(a) one can recognize that the three maxima in the SHG process correspond to three peaks of the scattering efficiency in figure 4(b). This behavior, neglecting possible absorption at the $\mathrm{SH}$ or the FF, can be intuitively explained by evaluating parameter $v=\left(Q_{\mathrm{FF}}\right)^{2} \times Q_{\mathrm{SH}} \times \zeta$, where $Q_{\mathrm{FF}}$ and $Q_{\mathrm{SH}}$ are the absorption and scattering efficiencies of the fundamental and second-harmonic modes, respectively, and $\zeta$ is the spatial overlap between the pump and the harmonic modes $[32,40]$. Thus, the conversion efficiency is expected to increase as $Q_{\mathrm{FF}}{ }^{2}$ because the intensity of the $\mathrm{SH}$ signal scales as the square of the intensity. From these observations, it is straightforward to understand that in order 
(a)

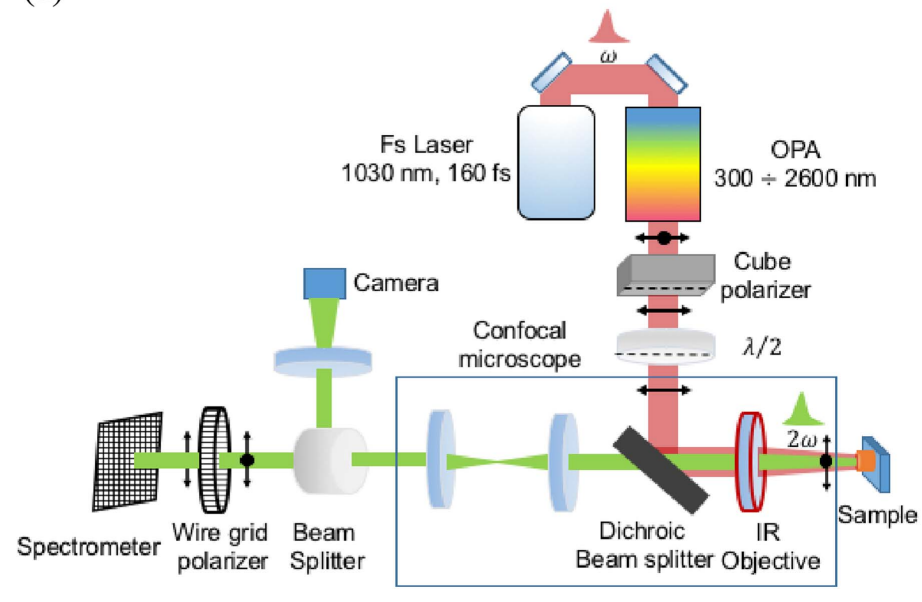

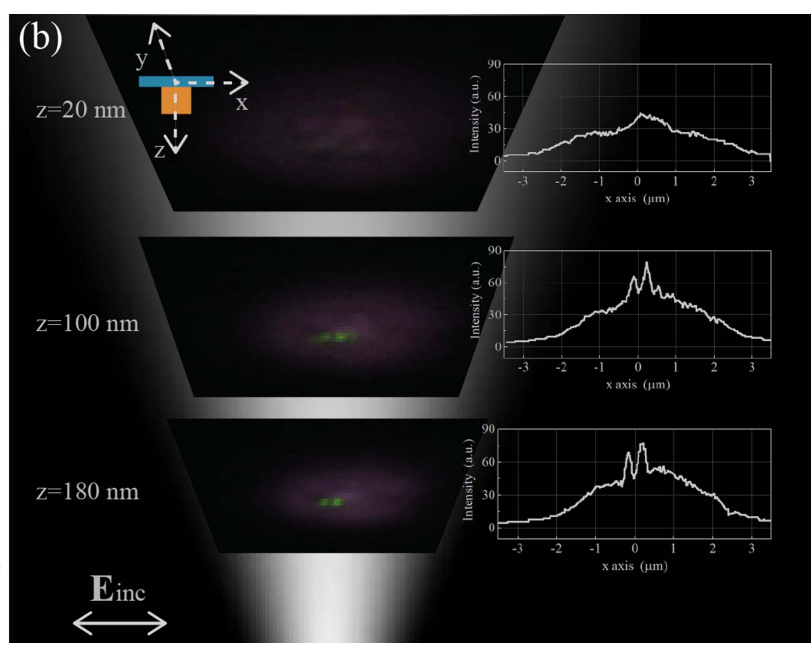

Figure 6. Experimental set-up for the SHG spectroscopy of individual nanoantennas. (b) Images of the radiated SHG at different focal planes along the antenna optical axis measured from a nanoantenna of radius $180 \mathrm{~nm}$ and height of $400 \mathrm{~nm}$ excited with a fundamental $1064 \mathrm{~nm}$ light. The incident field is polarized along the $x$-axis. The excitation spot, approximately $4 \mu \mathrm{m}^{2}$ in size, is centered at the individual nanoantenna. (Inset) Cross sections of the images along the $x$-axis.

to maximize $\eta_{\mathrm{SHG}}$, the cylinder geometry should, from one side, guarantee a fair tradeoff between the need of a high $Q_{\mathrm{FF}}$ and a high $Q_{\mathrm{SH}}$ to maximize the product $\left(Q_{\mathrm{FF}}\right)^{2} \times Q_{\mathrm{SH}}$ and, from the other side, support a mode at the $\mathrm{SH}$ wavelength with a good spatial overlap $\zeta$ with the pump mode $[36,40]$.

The SHG efficiency measured from the AlGaAs-on-AlOx nanoantennas for the fundamental wavelength of $1550 \mathrm{~nm} \mathrm{[33]}$ is shown in figure 5(b). The background SHG from the $\mathrm{AlO}_{\mathrm{x}}$ substrate is about three orders of magnitude below the SHG signal from the nanoantenna and was sub-tracted in the plot.

Three well-defined features can be clearly identified in the emission behavior at approximately $195 \mathrm{~nm}, 205 \mathrm{~nm}$ and 220 $\mathrm{nm}$. The calculated and measured radius-dependent behavior of the conversion efficiency reported in figure 5(b) singles out three resonances of the SHG nano-cavity modes excited at three specific radii with a maximum for $r=187 \mathrm{~nm}$. The agreement between numerical and experimental values confirms that a model resorting only to bulk nonlinear coefficients captures the main physics of the SHG process. The experimental data display an overall SHG collected signal proportional to the square of the pump intensity [33], which confirms the second-order origin of this process; the fact that the SHG spectral bandwidth is $\sqrt{ } 2$ times narrower than the excitation laser line (about $15 \mathrm{~nm}$ ) confirms that this signal stems solely from a coherent two-photon process.

The SHG spectra measured from the AlGaAs-on-AlOx nanoantennas of a radius of $225 \mathrm{~nm}$ is shown in figures 5(c), (d). (For these measurements, the experimental setup shown in figure 6(a) was used). Two resonances can be observed in the SHG spectral range at the fundamental wavelengths of approximately $1530 \mathrm{~nm}$ and $1670 \mathrm{~nm}$ for both co-polarized and cross-polarized configurations. Considering that in the experiment the pump wavelength was tuned with a step of 20 $\mathrm{nm}$, a very good agreement with the modelling predictions can be inferred with slightly larger discrepancies for the crosspolarised geometry. In particular, figure 5(d) shows that both simulations and experiments exhibit two SHG peaks; however, the relative amplitude of these peaks is not well reproduced by numerical data. This may be due to fabrication imperfections, which may affect the SHG efficiency. As a matter of fact, from the high-resolution transmission electron microscopy images, we can identify the presence of a random tilt (up to 10 degrees) in some nanopillars with respect to the axis normal to the surface, causing a local re-orientation of the susceptibility tensor at the individual nanoantenna level. This can be attributed to an asymmetric sinking of the nanopillars due to the substrate softening during the annealing step rather than to nanofabrication inaccuracies. The detailed investigation of this phenomenon, however, goes beyond the scope of this paper.

Both experimental data and simulations in figures 5(b)(d) show that the first two peaks, corresponding to smaller disk radii or longer wavelengths, have far-field SHG mainly copolarized with the pump beam, while the peak excited at lower wavelengths (or for bigger disk radii if working at a fixed wavelength) has a far-field SHG mainly cross-polarized with respect to the pump beam. The polarization properties of the generated SH signal is however very rich and deserve further investigations [48].

\section{SHG from AIGaAs nanoantennas: radiation pattern}

A typical radiation pattern at the second harmonic frequency (inset in figure 5(a)) illustrates the multipolar character of the $\mathrm{SH}$ source as well as the absence of SHG radiation along the axis of the cylinder. This is due to the symmetry of the structure and the symmetry of the $\chi^{(2)}$ tensor in zinc-blende crystals [60]. This is confirmed by investigating SHG from the individual AlGaAs antennas using a confocal microscope in reflection geometry as shown in figure 6(a). The light from an optical parametric amplifier (OPA) pumped with an 


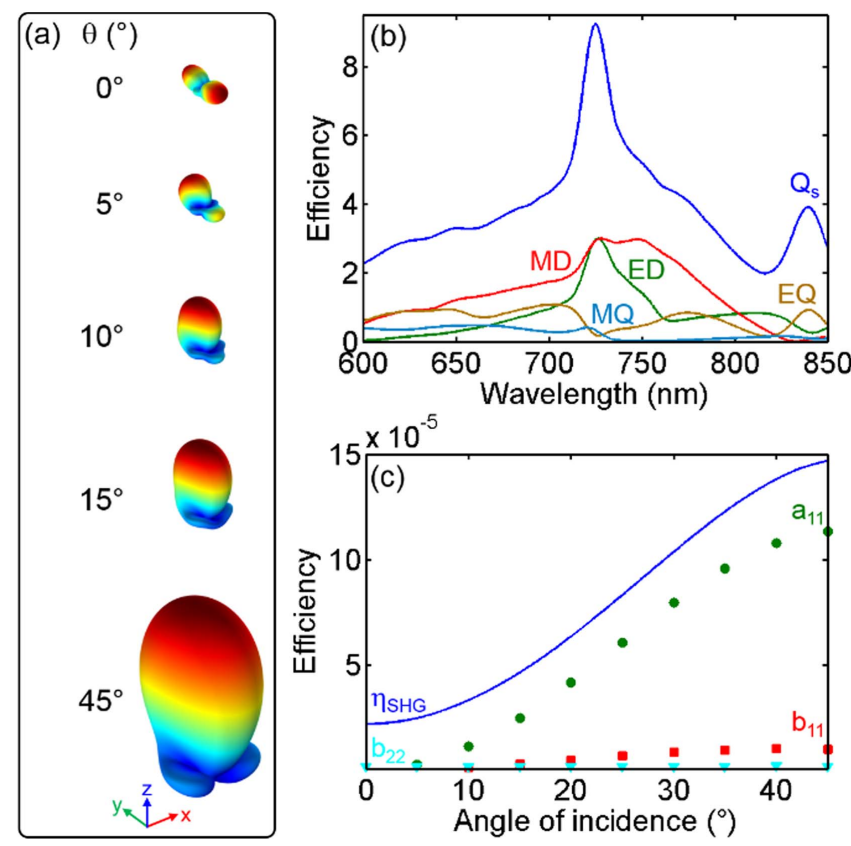

Figure 7. (a) Radiation patterns of the second harmonic generation for different incidence angles. (b) Linear scattering cross section spectra in the second harmonic frequency range for a nanoantenna with $r=187 \mathrm{~nm}$ and $h=400 \mathrm{~nm}$. The multipole decomposition of the scattering is also presented. (c) Second harmonic efficiency (continuous blue line) and its main multipolar contributions as a function of the incidence angle $\theta: a_{11}$ is the electric dipole (green dots); $b_{11}$ is the magnetic dipole (red squares), and $b_{22}$ is the magnetic quadrupole (cyan down triangles).

amplified ytterbium-doped potassium gadolinium tungstate (Yb:KGW) laser was used as a fundamental light in the SHG measurements. The fundamental light was tuned to $1064 \mathrm{~nm}$ and had a pulse duration of about $150 \mathrm{fs}$ and a repetition rate of $40 \mathrm{kHz}$, resulting in a constant average power of approximately $250 \mu \mathrm{W}$ at the sample. The set of polarisers was used to control polarization of the fundamental light. Figure 6(b) shows a microscope image of the SHG radiated from a nanoantenna of radius $180 \mathrm{~nm}$ and height $400 \mathrm{~nm}$ excited with polarization along the $x$-axis and at a fundamental wavelength of $1064 \mathrm{~nm}$. The antenna generates a bright green SHG signal. The substrate, instead, contributes with a photoluminescence (PL) signal centred at around $733 \mathrm{~nm}$ from the area illuminated by the excitation spot due to two-photon absorption in AlAs located beneath the $\mathrm{AlO}_{\mathrm{x}}$ layer [61]. The radiation diagram clearly shows two lobes in the $60^{\circ}$ lightcone accessible with the objective of $0.85 \mathrm{NA}$ in a $x-z$ plane above the antenna, as predicted by simulations [58]. An online supplementary movie from which a $z$-scan in figure 6 was plotted is also included, showing only PL or both SHG and PL radiation, depending on collection objective focus.

In many cases, the absence of the $\mathrm{SH}$ radiation in the direction of the nanoantenna axis is a drawback caused by the symmetry of the system. An effective strategy to overcome this is to break the symmetry using excitation at oblique incidence. Figure 7(a) shows the radiation pattern of the $\mathrm{SH}$ signal generated with an s-polarized pump at $\lambda=1445 \mathrm{~nm}$ from a cylinder with $r=187 \mathrm{~nm}$ and $h=400 \mathrm{~nm}$ for different angles of incidence of the fundamental beam. To understand the observed behavior, in figure 7(b) we show the scattering cross-section of this nanoantenna as well as the main multipolar contributions to the scattering process as a function of the pump wavelength. We can see that the antenna exhibits a strong SHG resonance of dominant magnetic and electric dipolar nature. The SHG efficiency and multipolar components of the total radiated $\mathrm{SH}$ field as a function of the incidence angle of the pump is shown in figure 7(c). With increasing incidence angle, the electric and magnetic dipolar contributions in the intensity of the $\mathrm{SH}$ scattering increase monotonically, leading to a radiation pattern that has a maximum along the cylinder axis. As the angle of incidence increases, the intensity of the SH light emitted in the normal direction increases, reaching a maximum at $\theta=45^{\circ}$, while the lobes in the other directions are progressively suppressed.

A similar behaviour is observed for the cross-polarized second harmonic generation process with the maximum efficiency for $r=225 \mathrm{~nm}$ at a fundamental wavelength of around $1500 \mathrm{~nm}$ (figure 5(b)). In figure 8, the fundamental wavelength is set to $\lambda=1570 \mathrm{~nm}$ and the incidence angle is varied from 0 to $45^{\circ}$. The intensity of the SH light emitted in the normal direction increases with the incidence angle, reaching a maximum at $\theta=45^{\circ}$, while the lobes in the other directions are suppressed. The reason for this behavior is that, by changing the incidence angle, the dipolar contribution in the SH scattering increases monotonically (lower panel in figure 8 ) in the same way as it was observed in figure 7(b) for the co-polarized $\mathrm{SH}$ peaks.

Figures 7 and 8 demonstrate that it is indeed possible to achieve directional $\mathrm{SH}$ emission using $\mathrm{AlGaAs}$ nanoantennas which might be very helpful for the design of nonlinear emitters [58].

\section{Outlook and perspectives}

The results that we reported here show the huge potential of the AlGaAs monolithic platform for nonlinear nanophotonics. To date, it is demonstrations focused on SHG enhancement in nanocylinders. However, they pave the way for other interesting second-order optical phenomena in nanostructures, such as sum- and difference-frequency generation. The former process (SFG) might also be used for classical-quantum correspondence experiments [62], while the latter (DFG) might prove useful for nanoscale optical parametric amplification [63]. This new monolitic platform provides innovative means for the design of on-chip tunable light sources, which are essential in many fields of modern photonics. Moving beyond the realm of nonlinear applications, AlGaAs might be a very promising material system for new metal-less metasurfaces. Indeed, absorption in plasmonic metasurfaces limits their performances in the near IR, and there is currently important research activity for finding new materials that might replace popular metals such as $\mathrm{Au}$ and $\mathrm{Ag}[64,65]$. Moreover, as discussed in section 2, AlGaAs can bring several advantages over $\mathrm{Si}$ for the future nanophotonic platforms. 


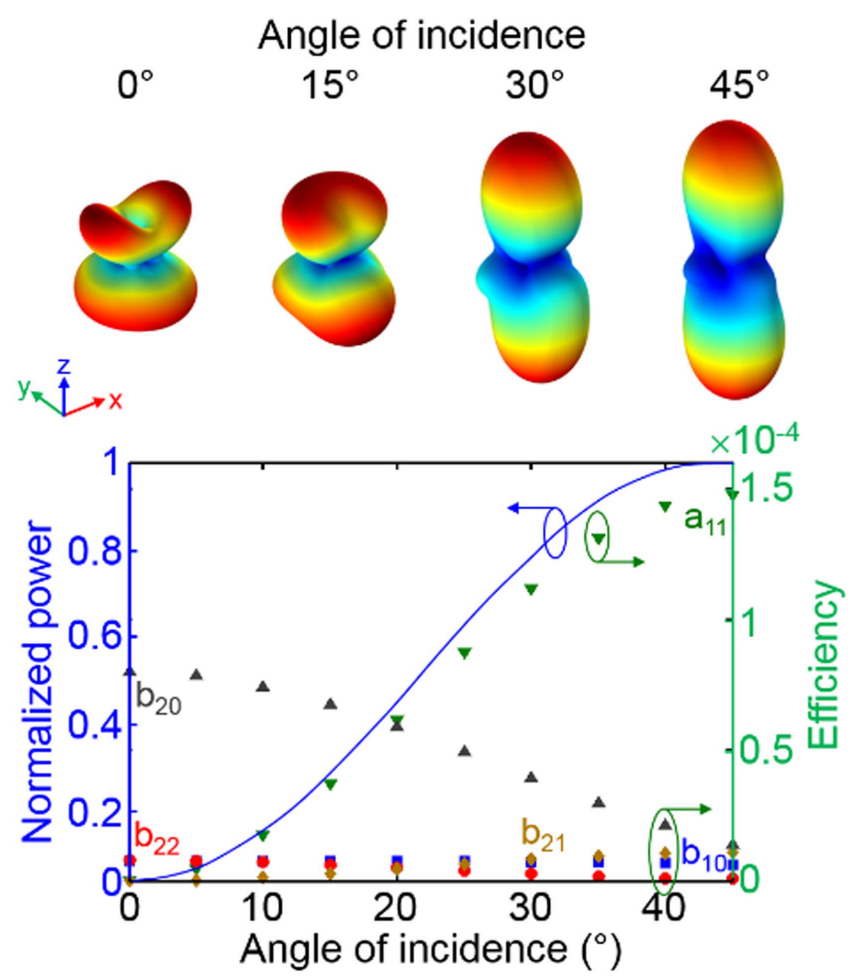

Figure 8. Upper panel: radiation pattern of the second harmonic generation for different incidence angles for the nanoantena with $r=225 \mathrm{~nm}$ and $h=400 \mathrm{~nm}$ and a fundamental wavelength $\lambda=1570 \mathrm{~nm}$. Lower panel: the normalized power emitted towards the positive $z$ direction (continuous blue line) and main multipolar contributions as a function of the incidence angle $\theta: a_{11}$ is the electric dipole (green downward triangles); $b_{10}$ is the magnetic dipole (blue squares); and $b_{20}, b_{21}$ and $b_{22}$ are the magnetic quadrupoles (grey upward triangles, ocher diamonds, and red dots, respectively).

This, in conjunction with tailored fabrication processes, will allow for the realization of three-dimensional structures using only planar lithography and etching procedures. This possibility increases the degrees of freedom in engineering AlGaAs-based metasurfaces beyond what is currently possible with the SOI platform.

\section{Conclusions}

We have overviewed recent results in the field of secondorder nonlinear optics at the nanoscale facilitated with alldielectric optical antennas. We have first presented the technological challenges and the proposed solutions for an AlGaAs-based monolithic platform for $\chi^{(2)}$ nonlinear nanophotonics. Then we have discussed the linear properties of all-dielectric nanoscale resonators, since efficiencies of nonlinear processes are significantly increased through the enhancement of the local fields due to the nanoparticle resonances. Having assessed the material platform, we have then reviewed the main reasons why these dielectric nano-objects offer such a high efficiency of $\chi^{(2)}$ effects, supporting the theoretical predictions with experimental results. In particular, we emphasized the role played by dipolar resonances at the fundamental frequency and multipolar resonances at the second harmonic wavelength. We have also discussed SHG directionality and shown some possible strategies to engineer the radiation pattern of nonlinear antennas, using as a tuning parameter the properties of the fundamental field, such as polarization state and/or propagation direction. Finally, we have shown theoretically that symmetry breaking with oblique excitation can steer the $\mathrm{SH}$ radiation main lobe in different directions, including along the nanoantenna axis.

\section{Funding sources}

This work and the contributions of all the authors has been supported in the framework of the Erasmus Mundus NANOPHI project, contract number 2013 5659/002-001. L Carletti, C De Angelis, A Locatelli, M Finazzi, L Ghirardini and $\mathrm{M}$ Celebrano acknowledge the support of CARIPLO (SHAPES-Second-HArmonic Plasmon-Enhanced Sensing). V F Gili acknowledges the 'Double Culture' PhD Grant of Sorbonne Paris Cité. G M and A V Z work was funded by ESPRC (UK) and the ERC iPLASMM project (321268). A V $\mathrm{Z}$ acknowledges support from the Royal Society and the Wolfson Foundation.

We thank Professor Dragomir Neshev for the useful discussions.

\section{References}

[1] Zhao J, Chremmos I D, Song D, Zhang P, Christodoulides D N, Efremidis N K and Chen Z 2015 Curved singular beams for three-dimensional particle manipulation Sci. Rep. 512086

[2] Haltout A M, Kildishev A V and Shalaev V M 2016 Evolution of photonic metasurfaces : from static to dynamic J. Opt. Soc. Am. B 33 501-10

[3] Yu N and Capasso F 2014 Flat optics with designer metasurfaces Nat. Mater. 13 139-50

[4] Agio M and Alù A 2013 Optical Antennas (Cambridge: Cambridge Universtiy Press)

[5] Minovich A E, Miroshnichenko A E, Bykov A Y, Murzina T V, Neshev D N and Kivshar Y S 2015 Functional and nonlinear optical metasurfaces Laser Photon. Rev. 9 195-213

[6] Smirnova D and Kivshar Y S 2016 Multipolar nonlinear nanophotonics Optica 3 1241-55

[7] Schuller J A, Barnard E S, Cai W, Jun Y C, White J S and Brongersma M L 2010 Plasmonics for extreme light concentration and manipulation Nat. Materials 9193

[8] Yu N, Genevet P, Kats M A, Aieta F, Tetienne J-P Capasso F and Gaburro Z 2011 Light propagation with phase discontinuities reflection and refraction Science 333-7

[9] Ni X, Emani N K, Kildishev A V, Boltasseva A and Shalaev V M 2011 Broadband light bending with plasmonic nanoantennas Science $\mathbf{3 3 5} 427$

[10] Ni X, Ishii S, Kildishev A V and Shalaev V M 2013 Ultra-thin, planar, babinet-inverted plasmonic metalenses Light Sci. Appl. 2 e72

[11] Aieta F, Genevet P, Kats M A, Yu N, Blanchard R, Gaburro Z and Capasso F 2012 Aberration-free ultrathin flat lenses and axicons at telecom wavelengths based on plasmonic metasurfaces Nano Lett. 12 4932-6 
[12] Chen W T et al 2014 High-efficiency broadband metahologram with polarization-controlled dual images Nano Lett. 14 225-30

[13] Balushi A A and Gordon R 2014 Label-free free-solution single-molecule protein-small molecule interaction observed by double-nanohole plasmonic trapping ACS Photonics 1389

[14] King M D, Khadka S, Craig G A and Mason M D 2008 Effect of local heating on the SERS efficiency of optically trapped prismatic nanoparticles J. Phys. Chem. C 11211751

[15] Albella P, de la Osa R A, Moreno F and Maier S A 2014

Electric and magnetic field enhancement with ultralow heat radiation dielectric nanoantennas: considerations for surfaceenhanced spectroscopies ACS Photonics 1 524-9

[16] Caldarola M, Albella P, Cortes E, Rahmani M, Roschuk T, Grinblat G, Oulton R F, Bragas A V and Maier S A 2015 Non-plasmonic nanoantennas for surface enhanced spectroscopies with ultra-low heat conversion Nat. Commun. 67915

[17] Staude I et al 2013 Tailoring directional scattering through magnetic and electric resonances in subwavelength silicon nanodisks ACS Nano 7 7824-32

[18] Decker M, Staude I, Falkner M, Dominguez J, Neshev D N, Brener I, Pertsch T and Kivshar Y S 2015 High-efficiency dielectric Huygens' surfaces Adv. Opt. Mater. 3 813-20

[19] Arbabi A, Horie Y, Bagheri M and Faraon A 2015 Dielectric metasurfaces for complete control of phase and polarization with subwavelength spatial resolution and high transmission Nat. Nanotechnology 1-8

[20] Chong K E et al 2016 Efficient polarization insensitive complex wavefront control using Huygens' metasurfaces based on dielectric resonant meta-atoms 3 514-9

[21] Jahani S and Jacob Z 2016 All-dielectric metamaterials Nat. Nanotechnol. 11 23-6

[22] Liu W, Miroshnichenko A E, Neshev D N and Kivshar Y S 2012 Broadband unidirectional scattering by magnetoelectric core-shell nanoparticles ACS Nano 6 5489-97

[23] Kauranen M and Zayats A V 2012 Nonlinear plasmonics Nat. Photonics 6737

[24] Keren-Zur S, Avayu O, Michaeli L and Ellenbogen T 2015 Nonlinear beam shaping with plasmonic metasurfaces $A C S$ Photonics 117-23

[25] Li G, Chen S, Pholchai N, Reineke B, Wong P W H, Pun E Y B, Cheah K W, Zentgraf T and Zhang S 2015 Continuous control of the nonlinearity phase for harmonic generations Nat. Mater. 14 607-12

[26] Wolf O et al 2015 Phased-array sources based on nonlinear metamaterial nanocavities Nat. Commun. 67667

[27] Ellenbogen T, Voloch N, Ganany-Padowicz A and Arie A 2009 Nonlinear generation and manipulation of Airy beams Nat. Photonics 3 395-8

[28] Noskov R E, Krasnok A E and Kivshar Y S 2012 Nonlinear metal-dielectric nanoantennas for light switching and routing New J. Phys. 14093005

[29] de Ceglia D, Vincenti M A, De Angelis C, Locatelli A, Haus J W and Scalora M 2015 Role of antenna modes and field enhancement in second harmonic generation from dipole nanoantennas Opt. Express 231715

[30] Kinsey N, DeVault C, Kim J, Ferrera M, Shalaev V M and Boltasseva A 2015 Epsilon-near-zero Al-doped ZnO for ultrafast switching at telecom wavelengths Optica 2 616-22

[31] Shcherbakov M R, Vabishchevich P P, Shorokhov A S, Chong K E, Choi D-Y, Staude I, Miroshnichenko A E, Neshev D N, Fedyanin A A and Kivshar Y S 2015 Ultrafast all-optical switching with magnetic resonances in nonlinear dielectric nanostructures Nano Lett. 15 6985-90

[32] Carletti L, Locatelli A, Stepanenko O, Leo G and De Angelis C 2015 Enhanced second-harmonic generation from magnetic resonance in AlGaAs nanoantennas Opt. Express 23 26544-50

[33] Gili V F et al 2016 Monolithic AlGaAs second-harmonic nanoantennas Opt. Express 2415965

[34] Liu S et al 2016 Resonantly enhanced second-harmonic generation using III-V semiconductor all-dielectric metasurfaces Nano Lett. 16 5426-32

[35] Celebrano $\mathrm{M}$ et al 2015 Emission engineering in germanium nanoresonators ACS Photonics 2 53-9

[36] Celebrano $\mathrm{M}$ et al 2015 Mode matching in multiresonant plasmonic nanoantennas for enhanced second harmonic generation Nat. Nanotechnology 10 412-7

[37] Thyagarajan K, Rivier S, Lovera A and Martin O J 2012 Enhanced second-harmonic generation from double resonant plasmonic antennae Opt. Express 2012860

[38] Navarro-Cia M and Maier S A 2012 Broad-band nearinfrared plasmonic nanoantennas for higher harmonic generation ACS Nano 63527

[39] Aouani H, Navarro-Cia M, Rahmani M, Sidiropoulos T P H, Hong M, Oulton R F and Maier S A 2012 Multiresonant broadband optical antennas as efficient tunable nanosources of second harmonic light Nano Lett. 124997

[40] Ginzburg P, Krasavin A, Sonnefraud Y, Murphy A, Pollard R J, Maier S A and Zayats A V 2012 Nonlinearly coupled localized plasmon resonances: resonant second harmonic generation Phys. Rev. B 86085422

[41] Smirnova D A, Khanikaev A B, Smirnov L A and Kivshar Y S 2016 Multipolar third-harmonic generation driven by optically induced magnetic resonances ACS Photonics 3 1468-76

[42] Shcherbakov M R et al 2014 Enhanced third-harmonic generation in silicon nanoparticles driven by magnetic response Nano Lett. 14 6488-92

[43] Maragkou M 2015 Dielectric nanostructures: ultrafast responses Nat. Mater. 141086

[44] Pu M, Ottaviano L, Semenova E and Yvind K 2016 Efficient frequency comb generation in AlGaAs-on-insulator Optica 3 $823-6$

[45] Dallesasse J M, Holonyak N, Sugg A R, Richard T A and El-Zein N 1990 Hydrolyzation oxidation of $\mathrm{Al}_{\mathrm{x}} \mathrm{Ga}_{1-\mathrm{x}} \mathrm{As}-$ AlAs-GaAs quantum well heterostructures and superlattices Appl. Phys. Lett. 572844

[46] Adachi S 1994 GaAs and Related Materials (Singapore: World Scientific Publishing)

[47] Person S et al 2011 Demonstration of zero optical backscattering from single nanoparticles ACS Nanoletters 131806

[48] Camacho-Morales R et al 2016 Nonlinear generation of vector beams from AlGaAs nanoantennas Nano Lett. 16 7191-7

[49] $\mathrm{Pu} \mathrm{M}$ et al 2016 Efficient frequency comb generation in AlGaAs-on-insulator Optica 3823

[50] Choquette K D et al 1995 Fabrication and performance of selectively oxidized vertical-cavity lasers IEEE Photon. Technol. Lett. 71237

[51] Savanier M et al 2013 Near-infrared optical parametric oscillator in a III-V semiconductor waveguide Appl. Phys. Lett. 103261105

[52] Scaccabarozzi L et al 2006 Enhanced second-harmonic generation in $\mathrm{AlGaAs} / \mathrm{Al}_{x} \mathrm{O}_{y}$ tightly confining waveguides and resonant cavities Opt. Lett. 313626

[53] Grahn P, Shevchenko A and Kaivola M 2012 Electromagnetic multipole theory for optical nanomaterials New J. Phys. 14 093033

[54] Gehrsitz S, Reinhart F K, Gourgon C, Herres N, Vonlanthen A and Sigg H 2000 The refractive index of $\mathrm{Al}_{\mathrm{x}} \mathrm{Ga}_{1-\mathrm{x}}$ As below the band gap: accurate determination and empirical modeling J. Appl. Phys. 877825

[55] Kuznetsov A I, Miroshnichenko A E, Fu Y H, Zhang J and Luk'yanchuk B 2012 Magnetic light Sci. Rep. 21 
[56] Evlyukhin A B, Novikov S M, Zywietz U, Eriksen R L, Reinhardt C, Bozhevolnyi S I and Chichkov B N 2012 Demonstration of magnetic dipole resonances of dielectric nanospheres in the visible region Nano Lett. 123749

[57] Rolly B, Stout B and Bonod N 2012 Boosting the directivity of optical antennas with magnetic and electric dipolar resonant particles Opt. Express 2020376

[58] Carletti L, Locatelli A, Neshev D N and Angelis C D 2016 Shaping the radiation pattern of second-harmonic generation generation from $\mathrm{AlGaAs}$ dielectric nanoantennas $A C S$ Photonics 3 1500-07

[59] Ohashi M, Kondo T, Ito R, Fukatsu S, Shiraki Y, Kumata K and Kano S S 1993 Determination of quadratic nonlinear optical coefficient of $\mathrm{Al}_{\mathrm{x}} \mathrm{Ga}_{1-\mathrm{x}}$ As system by the method of reflected second harmonics J. Appl. Phys. 74596

[60] Finazzi M, Biagioni P, Celebrano M and Duo L 2007 Selection rules for second-harmonic generation in nanoparticles Phys. Rev. B 76125414

[61] van der Ziel J P and Gossard A C 1978 Optical birefringence of ultra thin ALGaAs-GaAs multilayer heterostructures J. Appl. Phys. 49 2919-21
[62] Solntsev A S, Carletti L, Xu L, Poddubny A N, De Angelis C, Leo G, Neshev D N and Sukhorukov A A 2016 Quantumclassical correspondence for photon-pair generation in nonlinear dielectric nano-resonators Photonics and Fiber Technology 2016 (ACOFT, BGPP, NP) OSA Technical Digest (Optical Society of America) paper NT3A.4.

[63] Zhang Y, Manjavacas A, Hogan N J, Zhou L, Ayala-Orozco C, Dong L, Day J K, Nordlander P and Halas N J 2016 Toward surface plasmon-enhanced optical parametric amplification (SPOPA) with engineered nanoparticles: a nanoscale tunable infrared source Nano Lett. 163373

[64] Khorasaninejad M, Chen W T, Devlin R C, Oh J, Zhu A Y and Capasso F 2016 Metalenses at visible wavelengths: diffraction-limited focusing and subwavelength resolution imaging Science 3521190

[65] Liu S, Keeler G A, Reno J L, Sinclair M B and Brener I 2016 III-V semiconductor nanoresonators-a new strategy for passive, active, and nonlinear all-dielectric metamaterials Adv. Opt. Mater. 4 1457-62 